\title{
Experiencia interdisciplinar de desarrollo de videojuegos con alumnos de Bellas Artes e Informática
}

Ramón Mollá Vayáa, Francisco José Abad Cerdáb ${ }^{b}$ Boj Tovar, Clara ${ }^{c}$

a Departamento de Sistemas Informáticos y Computación, Universitat Politècnica de València - rmolla@dsic.upv.es b Departamento de Sistemas Informáticos y Computación, Universitat Politècnica de València - fjabad@dsic.upv.es y 'Departamento de Escultura, Universitat Politècnica de València - claboto@esc.upv.es.

\section{Abstract}

Multidisciplinary work groups have been established to develop video games involving two courses in the Faculty of Fine Arts and two courses in the Computer Science School.

The teams have followed the same methodology used in the video game development industry. They have used online applications to edit design documents collaboratively. They have shared code and other documents using GitHub. They have been collaborating using social networks. They have used the latest authoring and programming tools together with professional video game development environments. Agile methodologies have been used for the development of the projects. Progress monitoring meetings have been held throughout the four-month period.

Although many students had no previous experience developing video games nor using the engine studied in the course, the results have been very satisfactory. The average mark in each group has been close to 8 points. Students have positively assessed the pace followed and the follow-up sessions, the methodology used, the freedom to choose topics, working with students from other disciplines and the quality of the results.

\section{Keywords:}

Video games development, Unity, Fine Arts, multidisciplinary

\section{Resumen}

Se han establecido grupos de trabajo multidisciplina-res para desarrollar videojuegos que han implicado a dos asignaturas de la Facultad de BB.AA. y a otras dos de la Escuela de Ingeniería Informática.

Los equipos han seguido la misma metodología que se sigue en las empresas de desarrollo de videojuegos. Han utilizado aplicaciones en línea para editar los documentos de diseño de forma colaborativa, compartido código y otros documentos mediante GitHub, se han coordinado empleando redes sociales, han empleado herramientas de autor y de programación de última generación, junto con entornos profesionales de desarrollo de videojuegos. Se han empleado metodologías ágiles para el desarrollo de videojuegos y se han mantenido reuniones de control del progreso a lo largo de todo el cuatrimestre.

A pesar de que muchos alumnos no habian recibido docencia previa en videojuegos ni en la herramienta empleada en la asignatura, los resultados han sido muy satisfactorios. La nota media de cada especialidad ha estado cercana a los 8 puntos. Los alumnos han valorado positivamente el ritmo seguido y las sesiones de seguimiento, la metodología 
Palabras clave: Desarrollo de videojuegos, Unity, BB.AA., multidisciplinar

\section{Introducción}

Las competencias transversales son cada día más exigidas en los planes de estudios generales de las universidades (SÁNCHEZ-ELVIRA PANIGUA, 2010) y en concreto en las carreras de Informática (CAZORLA, 2010).

Aunque la nomenclatura para describirlas no está todavía normalizada, sí que existe un cierto consenso en cuáles son sus principales áreas (ALARCON, 2014). Tomando, por ejemplo, la clasificación realizada por la Universitat Politècnica de València, UPV, (ALVAREZ, 2016), en la Tabla 1 aparecen sus competencias transversales reconocidas:

Tabla 1. Competencias transversales reconocidas por la UPV.

\begin{tabular}{lc}
\hline Referencia & Nombre \\
\hline CT01 & Comprensión e integración \\
CT02 & Aplicación y pensamiento práctico \\
CT03 & Análisis y resolución de problemas \\
CT04 & Innovación, creatividad y emprendimiento \\
CT05 & Diseño y proyecto \\
CT06 & Trabajo en equipo y liderazgo \\
CT07 & Responsabilidad ética, medioambiental y profesional \\
CT08 & Comunicación efectiva \\
CT09 & Pensamiento crítico \\
CT10 & Conocimiento de problemas contemporáneos \\
CT11 & Aprendizaje permanente \\
CT12 & Planificación y gestión de tiempo \\
CT13 & Instrumental específica \\
\hline
\end{tabular}

Se observa que en la lista ofrecida, no aparece por ningún lado la competencia multidisciplinar (FRODEMAN, 2017). Esta competencia se menciona en bastantes informes y artículos que hablan de competencias transversales, sin embargo no suele concretarse. Esta competencia podría clasificarse como transtransversal dado que realmente es transversal a todas la competencias transversales.

Tradicionalmente se ha aplicado la competencia multidisciplinar de forma endogámica. Es decir, las asignaturas que la promueven, la implementan típicamente como trabajos compartidos entre diferentes asignaturas dentro de la misma especialidad (SERRANO TIERZ, 2013) (VERNET, 2011) y sin conexión con otras disciplinas externas (BLANCO, 2014)(PEÑA PAZ, 2011). Son pocas las experiencias capaces de plantearse la formación multidisciplinar excediendo los límites del centro o incluso de la propia universidad o país (CUADRADO, 2009).

\subsection{Proyectos multidisciplinares en docencia de videojuegos}

La creación de videojuegos es un área multidisciplinar en la que convergen informáticos, artistas, creativos, gestores, psicólogos o agentes de márketing y publicidad, entre otros. Los artistas son los encargados de generar los contenidos de autor y los informáticos, los que se encargan del desarrollo de código según las especificaciones del juego. 
Formar a estos profesionales conlleva no sólo el aprendizaje de las herramientas propias de su especialidad (herramientas de autor o de programación) sino también el aprendizaje de las herramientas propias del desarrollo e integración como son los entornos de desarrollo de los videojuegos (CHRISTOPOULOU, 2017).

Sin embargo, la creación de un videojuego es muy exigente en el uso de las competencias transversales sin las cuales es imposible acabar un producto de calidad. Todos los miembros del equipo de desarrollo de un videojuego deben de ser capaces de comprender y concretar adecuadamente especificaciones ambiguas o incompletas dadas por los creativos o los editores y ser capaz de integrar diferentes partes en una única visión global que abarque la totalidad de la historia que se desea experimentar (CT01), resolver muchos desafíos (CT03) diarios de índole práctica y a veces de forma coordinada entre diferentes especialistas (CT02). La naturaleza del videojuego obliga a realizar un análisis de requisitos (CT03) para después emplear el pensamiento sintético generando una solución óptima y eficiente dado que los videojuegos suelen trabajar con requisitos muy exigentes y en tiempo real. Este nivel de exigencia obliga a un aprendizaje permanente (CT11) en el uso de tecnologías punteras (CT13). Obliga a innovar soluciones, muchas veces de forma creativa (CT04) y coordinada entre todos los departamentos. Los videojuegos parten siempre de un diseño y se desarrollan siempre dentro de un proyecto (CT05) que tiene los tiempos y recursos marcados de forma férrea que obligan a una planificación y gestión de tiempo (CT12). Incluso en estudios pequeños de la escena indie se desarrolla siempre en equipos multidisciplinares que deben de ser guiados por el diseñador que es el que tiene la visión del videojuego (CT06) y en los que debe existir una fluida comunicación efectiva para que todos sus miembros tengan clara la idea del videojuego y se pueda comunicar esta a los publishers, rondas de financiación, clientes, márketing,... (CT08). Todos los videojuegos son catalogados por la normalizada etiqueta PEGI, que conlleva una marcada responsabilidad ética y profesional para cumplir con sus requisitos (CT07). En las fases finales del desarrollo del videojuego, durante la fase denominada Beta, tanto los desarrolladores como los testeadores del videojuego (beta-testers) deben de agudizar su pensamiento crítico con el fin de depurar y mejorar constantemente el producto (CT09). Los diseñadores deben proponer nuevas creaciones que compitan con el resto de la industria y eso les obliga a tener conocimiento permanentemente actualizado (CT10 y CT11).

Si bien algunas involucran más a unos departamentos u otros, normalmente la solución no puede ser tomada unilateralmente por ninguno de los departamentos dado que cualquier decisión asumida por los programadores tiene repercusiones estéticas audiovisuales o modificaciones de la historia y viceversa. Las decisiones adoptadas deben casar en la metáfora que desarrolla el videojuego y no debe distorsionar la visión global de la experiencia del jugador. La colaboración interdisciplinar es, por tanto, un requisito implícito, previo a las competencias transversales, sin la cual, esta industria no puede desarrollar sus productos.

En conclusión, reducir la formación en el desarrollo de un juego a una simple acumulación de contenidos o destrezas informáticas propias de su disciplina académica genera profesionales cualificados técnicamente pero no aptos para la industria.

En este contexto profesional, las competencias transversales de trabajo en equipos multidisciplinares no son un añadido cosmético más sino una cuestión de supervivencia del sector.

\section{Estado del arte}

La colaboración formal entre los estudiantes de arte y ciencias de la computación no es nueva (PARKER, 2004). Los proyectos multimedia conjuntos permiten desarrollar competencias transversales necesarias para la confección de aplicaciones reales en un entorno de trabajo lo más parecido al mundo real (CASTRO, 2018). Es más, el arte se puede llegar a emplear para introducir técnicas de computación a estudiantes en un contexto artístico (WOOD, 2016) como forma de facilitar su aprendizaje.

\subsection{Formación interdisciplinar en videojuegos}

En la enseñanza del desarrollo de videojuegos, existen tres aproximaciones:

1. Enfocada en los aspectos técnicos. Es el caso del Rochester Institute of Technology (RIT), el MIT (MIT) o la Drexel University (DU) que ofrecen un Bachelor y un Master of Science, sin formación complementaria artística.

2. Enfocada en los aspectos artísticos, de diseño o producción. Es el caso de Visual College of Art and Design of Vancouver (VCAD), la Vancouver Film School (VFS), Sheridan (Sheridan) o la 
Toronto Film School (TFS), la Autograf School (AS) en París, la Noroff School (NS) en Noruega, la universidad de Aalto (Aalto) o la Academy of Interactive Entertainment (AIE). Estos centros potencian la creación de narrativas, jugabilidad, modelado, arte conceptual, animación, efectos visuales, ... sin enfatizar tanto en aspectos de programación.

3. Mezcla de ambos tipos de formación creando equipos interdisciplinares. Es el caso de las universidades más afamadas en la formación para la creación de videojuegos, que suelen presentar titulaciones tanto de ciencia como de artes en su oferta de títulos. Así, aparece la University of Southern California (USC), el instituto DigiPen (DigiPen), que mantiene una fuerte relación con Nintendo o el Savannah College of Art and Design (SCAD); la University of Utah que ofrece los dos Bachelors anteriores y un único Master of Entertainment Arts \& Engineering (KESSLER, 2009) y mantiene una fuerte relación con la compañía EA. Esta universidad reúne estudiantes de la Escuela de Computación y de la División de Estudios Cinematográficos. El programa destaca por sus clases compartidas donde los estudiantes trabajan juntos y cooperan en proyectos de un año para realizar un videojuego o un corto animado desde cero. Otros ejemplos sobresalientes son la Carnegie Mellon University (CMU), el Champlain College (Champlain) o el Oulu Game LAB (Oulu) con un Bachelor of Computer Science y otro Bachelor of Arts.

\section{Objetivos}

El proyecto educativo PIME/19-20/167 cuyo título es "Experiencia en la organización de equipos multidisciplinares en asignaturas de creación de videojuegos", pretende generar Grupos Interdisciplinares de Trabajo, estables y permanentes, compuestos por alumnos con perfiles profesionales diferentes pero complementarios. En este caso, creativos e informáticos. El objetivo es que puedan trascender el marco de las asignaturas en los que se creen, no sólo mientras estas asignaturas están vivas, sino sobre todo, posteriormente, a su finalización, dando lugar a colaboraciones académicas más amplias y posteriormente profesionales. En este sentido, se pretende:

- Facilitar la generación de Start-ups e iniciativas de emprendimiento de cara a poder comercializar los productos generados en el último año de los estudios, tanto de la titulación de Diseño y Tecnologías Creativas (D.TT.CC.) como de Ingeniería en Informática.

- Producir trabajos académicos y TFGs de mayor valor añadido y producidos con una calidad más profesional.

- Evaluar la bondad de la colaboración realizada entre los alumnos.

- Generar una línea de trabajo que convierta los proyectos de asignatura en TFGs y éstos en productos comerciales que posteriormente puedan ser explotados bien directamente por los propios alumnos vía start-up o como producto adquirido por terceros.

- Generar un ambiente de trabajo lo más parecido a un entorno profesional para conseguir:

a. Preparar a los alumnos en el desarrollo de competencias transversales que necesitarán posteriormente en el desempeño de su actividad profesional.

b. Generar una experiencia de trabajo interdisciplinar REAL, no simulada.

c. Facilitar su integración en el entorno profesional mediante la creación de un portafolios de mayor calidad y profesionalidad.

\section{Descripción del proyecto educativo}

Si bien no existe en nuestra universidad un título universitario en videojuegos, sí que existen titulaciones que contienen asignaturas tanto de diseño, como de desarrollo de contenidos o de programación de videojuegos.

Este proyecto puso en contacto a alumnos del grado en Ingeniería Informática que se imparte en la Escuela Técnica Superior de Ingeniería Informática (ETSIINF) y el grado en Diseño de Tecnologías Creativas (D.TT.CC.) que se imparte en la Facultad de BB.AA.

Las cuatro asignaturas participantes en esta experiencia se describen a continuación. El resumen cronológico de su impartición a lo largo de tercer y cuarto curso y según se imparte en un semestre u otro puede verse en la Tabla 2. 
Tabla 2 Cronograma de la impartición de la docencia en las asignaturas involucradas en el proyecto educativo

\begin{tabular}{cccc}
\hline Titulación & \multicolumn{3}{c}{ Curso / Semestre } \\
\hline & $3^{\circ} / 6$ & $4^{\circ} / 7$ & $4^{\circ} / 8$ \\
D.TT.CC. (BB.AA.) & $\operatorname{DiV}[1] 9 \mathrm{c}(2 \mathrm{D})$ & $\operatorname{DeV} 9 \mathrm{c}$ (3D) & \\
I.I. (ETSIInf) & & IPV 4.5c (3D) & EdV 4.5c (2D) \\
\hline
\end{tabular}

\section{ETSIInf}

Existen dos asignaturas optativas de cuarto curso del grado en Ingeniería Informática que tienen una carga de 4.5 créditos cada una, haciendo un paquete formativo en videojuegos de 9 créditos en total. Se imparten en:

- Primer cuatrimestre. Introducción a la Programación de Videojuegos (IPV) introduce los fundamentos de la disciplina y se forma a los alumnos en el uso de la programación de videojuegos.

- Segundo cuatrimestre. Entornos de Desarrollo de Videojuegos (EDV) incorpora las lagunas que no se han podido cubrir en IPV ofreciendo al alumno un temario completo y autocontenido en cada asignatura y a la vez complementario entre sí.

$B B . A A$.

Existen dos asignaturas optativas con una carga de 9 créditos cada una, haciendo un paquete formativo de 18 créditos en total. Se imparten en:

- Tercer curso ( $2^{\circ}$ cuatrimestre). Diseño de Videojuegos (DiV) introduce al alumno en la elaboración de los contenidos, reglas de juego, jugabilidad, narrativa, ... que determina el contenido de un juego. El objetivo es realizar un prototipo inicial muy sencillo del videojuego.

- Cuarto curso (1er cuatrimestre). Desarrollo de Videojuegos (DeV) recoge la formación recibida de diseño de videojuegos y genera al final de cuatrimestre un MVP (RIES, 2011) y al menos un par de niveles de juego completos.

La planificación temporal de estas asignaturas puede verse en el siguiente esquema.

\subsection{Integración}

Las asignaturas adscritas a la Facultad de BB.AA. van dirigidas a un alumnado cuyas competencias se centran en el uso de herramientas de autor orientadas a crear los contenidos de las producciones multimedia. Sus competencias en desarrollo de programas son limitadas.

Por otro lado, los alumnos de la ETSINF que eligen las asignaturas optativas en videojuegos provienen de un perfil fundamentalmente técnico y, salvo contadas excepciones, cuentan con escasos conocimientos en la creación de contenidos multimedia en general.

Es por ello que el alumnado de ambas titulaciones es bastante compatible entre sí y podría dar lugar a fuertes sinergias si se les diera la oportunidad de colaborar entre ellos.

En este apartado se relatan las dificultades que aparecieron desde el punto de vista de la integración y coordinación de todas las asignaturas en un proyecto común, así como los modos en los que se resolvieron.

\subsubsection{Tecnología}

Construir videojuegos actualmente es más sencillo que hace unos años debido principalmente a la existencia de entornos de desarrollo de videojuegos que facilitan la migración de aplicaciones entre diversas plataformas de una forma bastante sencilla.

De entre todos los motores que actualmente se pueden emplear para este desarrollo, se destacaría a Unity (Unity) por su facilidad de uso, la enorme variedad de plug-ins que se pueden insertar en el entorno para poder ampliar su capacidad de desarrollo, el precio que presenta tanto para poder emplear sus licencias de uso, como el coste medio que suelen presentar los plug-ins desarrollados por terceros y el bajo coste de aprendizaje.

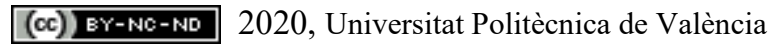

Congreso In-Red (2020) 
Todas estas características le convierte en un candidato ideal para comenzar el desarrollo de videojuegos por parte de los estudiantes, así como para el desarrollo de entornos de simulación, serious games, etc. De hecho, es la herramienta más utilizada por la industria de los videojuegos de acuerdo con los últimos datos consultados en los populares sitios de referencia gamasutra o itch.io (Gamasutra).

Es por ello que todas las asignaturas descritas se han tenido que unificar tecnológicamente empleando el entorno de desarrollo Unity.

Esto ha obligado a cambiar totalmente los contenidos, temario y metodologías de la asignatura IPV. Esta asignatura empleaba el entorno de desarrollo MS-Visual Studio y el lenguaje C++, el cual ha tenido que cambiarse a Unity y C\# si bien ha mantenido MS-Visual Studio como entorno de desarrollo de código.

\subsubsection{Contenidos}

Los contenidos de la asignatura DiV están coordinados con los de DeV y los de IPV con los de EDV. Además, los contenidos de DeV e IPV, están coordinados entre sí para implementar los trabajos multidisciplinares. Por lo tanto, los contenidos de las cuatro asignaturas son interdependientes entre sí porque cualquier modificación de los contenidos de una asignatura altera los de la coordinada e indirectamente al resto. Por lo tanto, deben de verse como un todo monolítico si bien a caballo de tres cursos y dos titulaciones diferentes. Véase la Tabla 2.

\section{Diseño de Videojuegos}

Esta asignatura, de tercer curso de BB.AA., se creó el curso pasado. Se centra fundamentalmente en aspectos teóricos y metodológicos del diseño niveles, personajes, historias, reglas e interfaz de juego,...

La idea es que aquellos alumnos que se hubieran decantado por la rama de videojuegos, hubieran tenido la experiencia de desarrollar al menos un prototipo de juego en 2D y otro en 3D antes de finalizar sus estudios. Dado que desarrollar un juego en 2D es tecnológicamente más sencillo que hacerlo en 3D, se decidió desarrollar la parte 2D en tercero y la de 3D en cuarto, en la asignatura de Desarrollo de Videojuegos.

En el caso de la asignatura de DiV, se introduce a los alumnos en el funcionamiento de Unity y en el lenguaje de programación que utiliza $(\mathrm{C \#})$, insistiendo sobre todo en aquellos aspectos que diferencian a Java (lenguaje empleado en cursos anteriores) de C\#. Téngase en cuenta que los alumnos que las cursan, disponen de conocimientos rudimentarios de años anteriores en programación en Java en el entorno Processing y de JavaScript.

Por otro lado, no importa tampoco que no alcancen un dominio de ambas tecnologías (Unity y C\#) dado que Unity se emplea como un prototipador rápido para analizar la viabilidad de la idea del videojuego, más que para desarrollarlo.

\section{Desarrollo de Videojuegos}

$\mathrm{DeV}$, de cuarto curso de BB.AA., se creó en el presente curso. Más orientada a aspectos de creación e integración de contenidos de autor como fondos, selección de colores, creación del aspecto visual de los personajes y elementos del juego, música, guionado, alguna programación sencilla e integración.

Una parte de los alumnos matriculados puede haber cursado también la asignatura de Diseño de Videojuegos, pero no es un prerrequisito hacerlo, Es por ello que que no se puede garantizar una homogeneidad de alumnado al ser ambas asignaturas optativas.

Puede darse la circunstancia de que ambas sean elegidas por alumnado Erasmus e incluso que estos alumns sean diferentes. De hecho ocurrió que hubo alumnos que eligieron una y no otra e incluso que eligieron las dos, dándose la circunstancia de que se cursaran en orden inverso al recorrido por los alumnos nativos debido a que la asignatura de cuarto se imparte en el primer cuatrimestre y la de tercero en segundo.

Se dió la circunstancia de que alumnos que habían cursado la asignatura de tercero no pudieron elegir la de cuarto por encontrarse en el primer cuatrimestre de estancia Erasmus en el extranjero. Razón por la cual, sólo la mitad de los alumnos matriculados en la asignatura de cuarto no habían cursado la de tercero. Estos alumnos venían sin experiencia previa de Unity y por lo tanto de C\#. Así mismo, presentaban un dominio menor de la programación.

La disparidad de nivel de entrada se resolvió suministrando material docente de la asignatura de DiV a los alumnos que no la habían cursado (Erasmus o no) para que se estudiaran por su cuenta los conceptos básicos que se asumían ya conocidos, en un intento de nivelación de los conocimientos de los alumnos. 
Por otro lado, se asumió que los alumnos no conocían el entorno de desarrollo y se realizó una introducción rápida al entorno de programación y al lenguaje. Esto sirvió de repaso a los alumnos provenientes de DiV y de cursillo acelerado para los que desconocían la herramienta.

A la hora de crear los grupos de trabajo para el desarrollo de los videojuegos, se intentó que en cada grupo hubiera al menos un miembro con conocimientos de la teoría de diseño de videojuegos.

\section{Introducción a la Programación de Videojuegos}

Los contenidos del curso pasado de esta asignatura que se imparte en la ETSIInf se tuvieron que eliminar completamente. Se tuvo que generar contenidos totalmente nuevos, como si la asignatura fuera de nueva creación. Está orientada a aspectos más informáticos del desarrollo de videojuegos como la programación, sincronización de efectos y sonidos, uso de shaders, simulación física, inserción de plugins, animación de estados, integración,...

Sus contenidos se cambiaron en función de los impartidos en la asignatura de Desarrollo de Videojuegos y se tuvo en cuenta la temporización para que estuvieran sincronizados entre ellos.

\section{Entornos de Desarrollo de Videojuegos}

Los contenidos 3D que se impartían en el curso pasado en esta asignatura se tuvieron que adaptar o incluso trasvasar a la asignatura IPV porque tanto en la titulación de BB.AA. como en la de la ETSIInf, se quería que los alumnos que optaran por las dos asignaturas de videojuegos, hubieran desarrollado al menos un videojuego en $2 \mathrm{D}$ y otro en $3 \mathrm{D}$.

Por eso, dado que en BB.AA. la secuencia estaba marcada entre DiV (2D) y DeV (3D), no hubo más remedio que impartir IPV en 3D y EDV en 2D aunque la secuencia en informática debería haber sido al revés, tal y como se realizó en BB.AA.

Se adaptaron también los contenidos de EDV con el fin de incorporar a la asignatura las lagunas que no se pudieron cubrir en IPV. El objetivo fue hacer un temario completo y autocontenido en cada asignatura y a la vez complementario entre sí, de forma que los alumnos que hubieran realizado primero la asignatura de IPV, tuvieran el mínimo solape de contenido.

\subsubsection{Metodología}

Todas las asignaturas coordinadas en el proyecto educativo desarrollan un producto final en grupos de trabajo mediante un Aprendizaje Basado en Problemas (PBL).

El objetivo de la asignatura $\mathrm{DiV}$ es generar toda la documentación asociada a las diferentes fases del diseño de un videojuego: concept, pitch-doc y Game Design Document (GDD), así como desarrollar un prototipo básico (MVP) que muestre la viabilidad de la idea del videojuego.

En el caso del resto de asignaturas, todos los alumnos siguen la misma metodología que se emplea en la industria de los videojuegos. Así, se genera primero el concepto de juego y después se elaboran los pitchdocs. Se votan las ideas y se eligen las mejores propuestas. Estas propuestas se desarrollan en formato GDD y finalmente se confecciona el videojuego 2D en EDV y 3D en las asignaturas multidisciplinares: IPV y DeV.

Tanto DiV como EDV no se coordinan con ninguna otra asignatura mientras se imparten. Sus proyectos son autocontenidos y no son multidisciplinares. Los grupos suelen ser alrededor de tres personas, todas pertenecientes a la misma titulación y grupo.

IPV y DeV coinciden en el tiempo (primer cuatrimestre), en el objetivo de las asignaturas, en el curso en el que se imparten y son las que se han coordinado entre sí para poder trabajar en equipos multidisciplinares. Este año se matricularon 33 estudiantes de la asignatura de IPV y 14 personas de DeV. Dado que los alumnos de BB.AA. se encontraban más cómodos en grupos en los que hubieran al menos dos diseñadores, se establecieron 7 grupos de trabajo, lo cual obligaba a que hubieran inicialmente entre 4 y 5 alumnos de informática en cada grupo de trabajo.

\subsubsection{Evaluación}

Se han realizado tres tipos de actos de seguimiento, algunos de ellos evaluables:

- Entregas: consistente en la subida de documentación escrita a la plataforma web de la asignatura. En concreto, los pitch doc y GDDs en sus diferentes versiones.

- Defensa: consistente en una presentación frente al resto de compañeros en presencia del profesorado de la asignatura.

- Examen: prueba escrita de tipo test sobre los contenidos teóricos de la asignatura ( $10 \%$ de la nota final) y prueba de manejo de la herramienta frente al ordenador $(15 \%)$. 
La Tabla 3 muestra un cronograma de los actos de seguimiento que se realizaron durante las asignaturas, junto al peso del acto de evaluación en la nota final, si procede.

\section{Entregas}

El primer acto de seguimiento en la asignatura consistió en la entrega del Pitch-Doc. En el campo de los videojuegos, es común presentar la idea de un nuevo juego mediante un documento corto que enumera las principales características del juego (tipo de juego, personajes, mecánicas principales, etc.). De esta forma, cada alumno tuvo que preparar un breve documento exponiendo el proyecto de un juego original. Uno de los objetivos de la asignatura es que los trabajos de la asignatura surgieran como proyectos propuestos por los propios alumnos, que votarían a las mejores ideas, y seleccionaran en qué proyecto les gustaría participar. Esta primera entrega se planteó para realizar un primer filtrado de proyectos que no fueran viables a priori, o no tuvieran la entidad requerida. Finalmente no se filtró ningún trabajo.

\section{Defensas}

El siguiente acto evaluable consistió en una defensa del Pitch-Doc. Se recogieron las presentaciones a priori y se organizaron asignando un identificador a cada alumno para agilizar al máximo esta sesión.

En una sesión de trabajo en la que estaban convocados todos los alumnos de ambas asignaturas, cada alumno tenía 3 minutos como máximo para presentar su idea. Aquellos que se excedieron, fueron interrumpidos.

Tabla 3. Cronograma de los actos de seguimiento, junto a su peso en la calificación final.

\begin{tabular}{|c|c|c|}
\hline Acto & Sem & Evaluación \\
\hline Entrega Pitch-Doc & 2 & No evaluable \\
\hline Defensa Pitch-Doc & 3 & $\begin{array}{c}\text { Co-evaluación defensa }+ \\
\text { contenido } 5 \%+5 \%\end{array}$ \\
\hline Defensa GDD & 5 & Evaluable 5\% \\
\hline $\begin{array}{l}\text { Defensa preliminar } \\
\qquad 1^{\text {er }} \text { nivel }\end{array}$ & 8 & $\begin{array}{l}\text { No evaluable. Críticas, } \\
\text { sugerencias, comentarios }\end{array}$ \\
\hline Defensa 1er nivel & 11 & Evaluable. $15 \%$ \\
\hline Feria de proyectos & 12 & $\begin{array}{l}\text { Co-Evaluación externa, } \\
\text { tests de usuarios }\end{array}$ \\
\hline Examen de teoría & 12 & $\begin{array}{l}\text { Evaluable. Tipo test. } \\
\qquad 10 \%\end{array}$ \\
\hline $\begin{array}{l}\text { Examen de } \\
\text { prácticas }\end{array}$ & 13 & Evaluable. 15\% \\
\hline $\begin{array}{l}\text { Defensa final y } \\
\text { GDD definitivo }\end{array}$ & 15 & Evaluable. $35 \%+10 \%$ \\
\hline
\end{tabular}

Aparte de defender su idea, en esta sesión cada alumno tenía que seleccionar hasta 4 proyectos (no incluyendo el suyo propio) y evaluar tanto la idea del juego como la presentación de sus compañeros mediante un formulario basado en una escala de Likert. Puede verse el formulario de evaluación utilizado en la Fig. 1.

Para realizar las votaciones se empleó material impreso en papel con el fin de facilitar la velocidad de votación y agilizar el proceso de recogida de evaluaciones. Posteriormente se volcaron las opiniones en hojas de cálculo que sirvieron para generar una primera coevaluación entre alumnos.

De los trabajos más votados, se eligió un total de 7 por las cuestiones logísticas explicadas anteriormente. Sobre esa elección, se montaron los grupos de desarrollo multidisciplinares. Estos grupos crearon los documentos de diseño del videojuego o GDD a partir de plantillas que se tenían que adaptar a las características de cada proyecto. 
Cada acto de defensa del proyecto a lo largo del curso se acompañaba de una nueva versión actualizada (Entrega) del GDD. Los GDDs se basaron en plantillas que se adaptaron a las características de cada propuesta.

\section{Elevator pitch}

\section{Nombre:}

\section{Número:}

Evalúa, por una parte, la idea del juego (si piensas que será divertido, si la idea está bien desarrollada, si piensas que se puede implementar en la duración de la asignatura). Evalúa también la presentación (el material ha servido para entender el juego, el discurso ha sido fácil de entender, piensas que ha estado bien preparado, etc.)

\begin{tabular}{|c|c|c|c|c|c|c|c|c|c|c|}
\hline & \multicolumn{5}{|c|}{ Idea del juego } & \multicolumn{5}{|c|}{ Presentación } \\
\hline Juego & $=$ & $\because$ & $\because$ & $\because$ & $\because$ & $=$ & $\because$ & $\cdots$ & $\because$ & $\because$ \\
\hline 1 & & & & & & & & & & \\
\hline 2 & & & & & & & & & & \\
\hline 3 & & & & & & & & & & \\
\hline
\end{tabular}

Fig. 1 Formulario de evaluación de las presentaciones de las propuestas de proyectos

En las presentaciones públicas o defensas, cada grupo presentó el estado de su proyecto al resto de los alumnos de ambas asignaturas. En dicha defensa se mostraban los avances realizados desde la presentación anterior (ver Fig. 2). Al finalizar cada presentación, los profesores comentaban los puntos fuertes, los fallos de diseño detectados y se sugerían ampliaciones. Era común que compañeros aportaran alguna idea o preguntaran sobre cómo se había resuelto algún problema técnico.

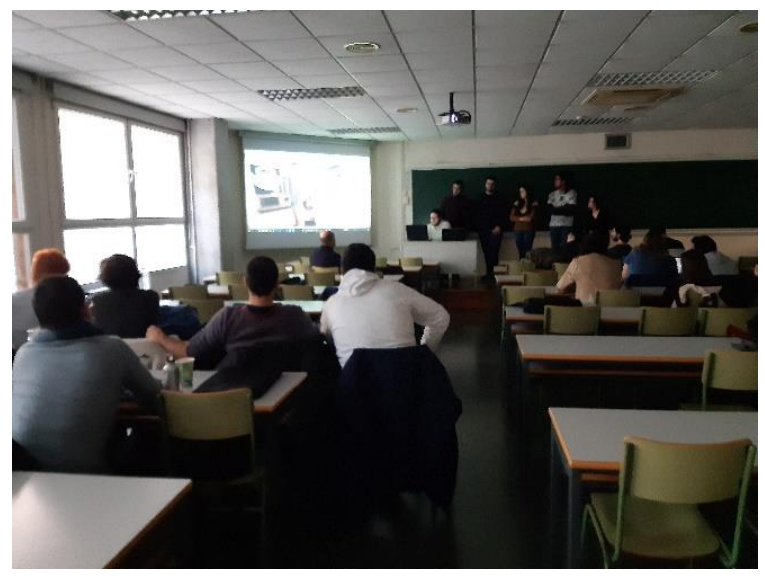

Fig. 2 Un momento de una de las presentaciones del estado de los trabajos

Uno de los actos de defensa se realizó en el marco de la II Feria de Proyectos realizada en el hall de la ETSIInf, y a la que podía acudir cualquier persona a ver los proyectos que se estaban realizando por parte de sus propios compañeros. En esta jornada, de asistencia voluntaria, los grupos tuvieron la posibilidad de mostrar una versión preliminar de su juego. Dicha versión se correspondía principalmente con un sistema de menú y un primer nivel completamente jugable. Los grupos eran conscientes de la oportunidad de mostrar su juego a terceras personas, prepararon encuestas para los asistentes y tomaron notas de las críticas y de las sugerencias de mejora.

La Fig. 3 muestra un momento de las presentaciones del trabajo final. 


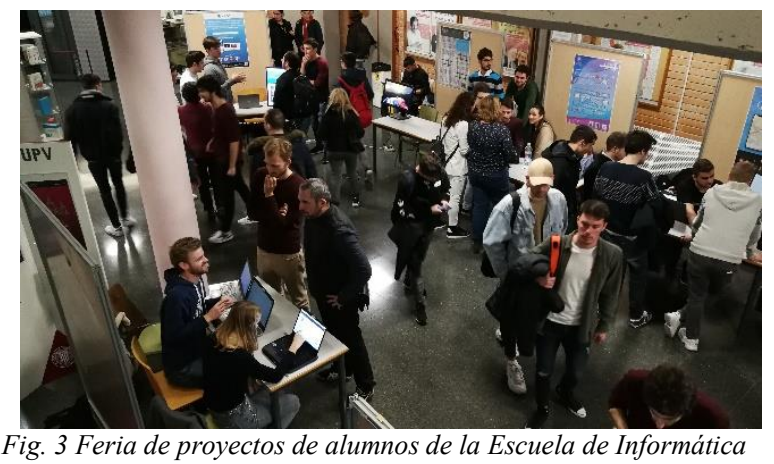

En la presentación final, los grupos tuvieron la oportunidad de mostrar los avances realizados desde la Feria de proyectos. Todos los equipos presentaron al menos dos niveles de juego completos y muchos de ellos incluyeron sugerencias recibidas en la Feria.

Por cada defensa evaluable de trabajo grupal se realizó una encuesta empleando herramientas internas de la intranet de la asignatura en la que cada alumno debía de valorar la actividad desarrollada por cada miembro de su equipo y a sí mismo. Se utilizó un cuestionario donde los miembros del grupo podían evaluar el grado de implicación de cada miembro en el trabajo realizado para el entregable mediante una escala de Likert de 5 niveles (Nada, Poco, Normal, Bastante y Mucho). Otra forma de corroborar el grado de implicación de los miembros de cada equipo consistió en la realización de un examen práctico de Unity en el laboratorio.

Un grado de implicación alto se tenía que traducir en un dominio elevado de la herramienta y, por lo tanto, la nota práctica debía estar en consonancia.

\section{Resultados}

Cada uno de los 7 grupos completó un juego con, al menos, dos niveles completamente desarrollados: jugabilidad completa, objetivos claros, navegación por menús e interfaz básico de juego. El contenido de autor de todos los juegos es original y estuvo desarrollado por los alumnos de la Facultad de Bellas Artes si bien, puntualmente se incorporó contenido externo procedente de la tienda en línea de Unity. Véase de la Fig. 4 a la Fig. 7. La programación del juego fue realizada principalmente por los alumnos de Informática y algo de ayuda de los alumnos de BB.AA.

\subsection{Emprendimiento}

El objetivo del proyecto educativo era que, al realizarse los proyectos en el primer cuatrimestre del curso, los alumnos que así lo desearan, pudieran continuar con el proyecto ya arrancado en forma de TFG, bien individualmente, bien colectivamente con el fin de seguir desarrollando la idea. Al acabar el TFG, se debería determinar si valía la pena convertir el videojuego en un producto comercial real tras un año de trabajo en el mismo.

Había completa libertad para poder elegir cualquier opción:

- $\quad$ El TFG podría basarse en trabajos en los que hubiera colaborado el alumno o en trabajos de compañeros.

- $\quad$ El TFG podría ser individual o colectivo, integrado por cualquier combinación de alumnos, tanto de BB.AA. como de informática.

En este sentido, habría que hacer un reconocimiento de derechos de autor según los contenidos generados, tanto si se presentaba el trabajo como TFG o se acababa comercializándolo.

Al acabar las asignaturas se realizó una encuesta online sobre la experiencia con el fin de obtener información acerca de posibles mejoras para cursos siguientes. De los 18 alumnos que contestaron, un $72.2 \%$ expresó su intención de realizar un TFG en videojuegos. De ellos, la mitad, utilizarían el trabajo de la asignatura como base. Por otra parte, un tercio tenía la intención de comercializar su videojuego. 

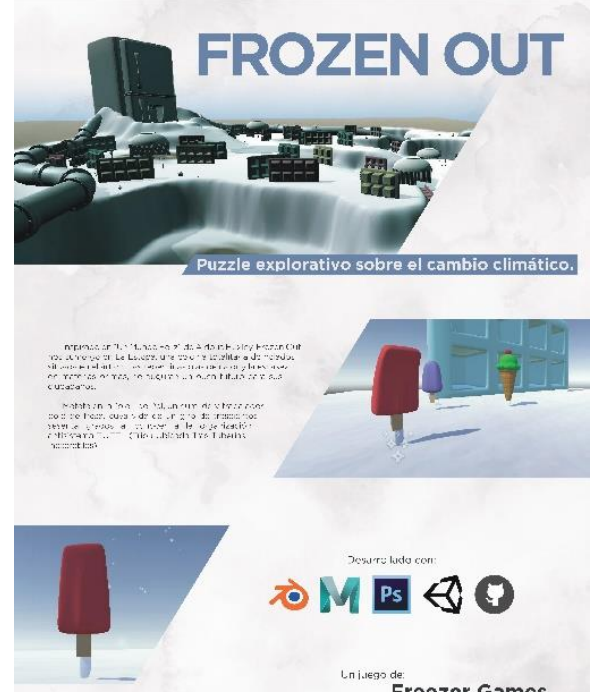

$\gg M P S O$

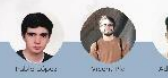

Lni.tego de Freezer Games

Fig. 4 Póster del demostrador del videojuego Frozen Out presentado en la II Feria de Proyectos de la ETSIINF

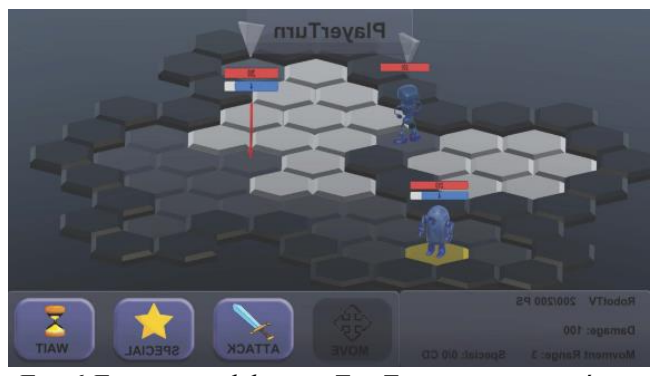

Fig. 6 Fotograma del juego Toy Tactics en ejecución

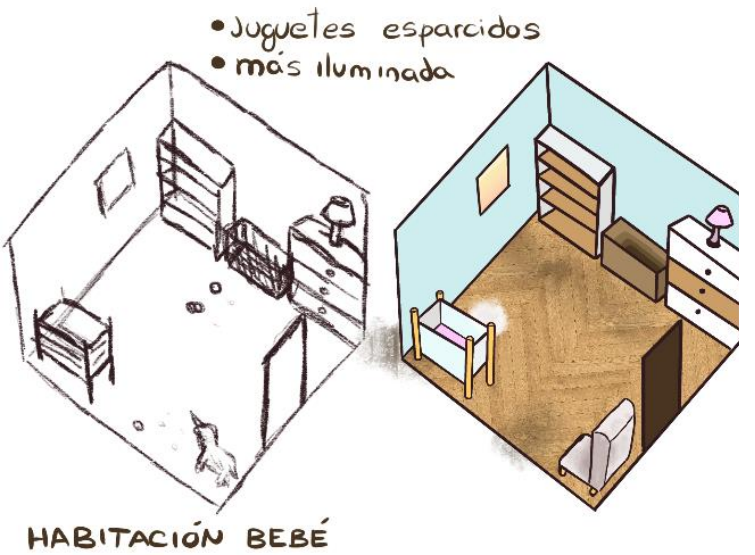

Fig. 5 Concept art del primer nivel del juego Amigurumi
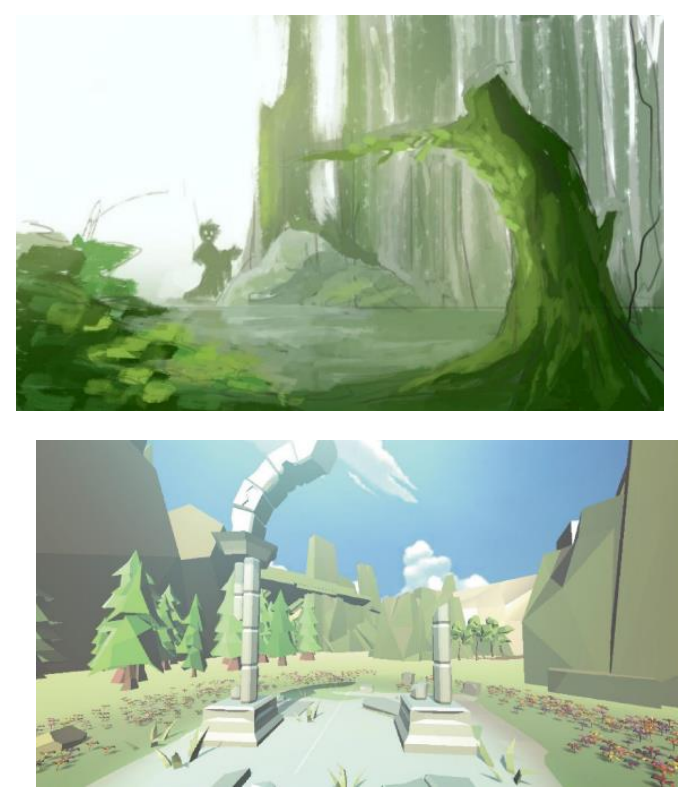

Fig. 7 Concept art y fotograma del juego de Will-o'-the-Wisp

\subsection{Seguimiento}

En total, hubo 6 actos de defensa, incluyendo la Feria de proyectos de la ETSINF. De media hubo, al menos, un acto de seguimiento mensual del proyecto, siendo al principio (Pitch-Doc y GDD) y al final cuando estos actos se duplicaron. Un calendario tan denso de defensas ha tenido diversos resultados:

- El arranque de los proyectos fue rápido al principio por la celeridad de los plazos de presentación de las propuestas de diseño: pitch-docs y GDD.

- La reunión mensual facilitó el seguimiento del proyecto por parte de los profesores y aseguró una evolución adecuada: se corrigieron desviaciones y malas prácticas, se realizaron sugerencias de mejora,...

- Aumentar la motivación y grado de implicación de los miembros del equipo.

- Mantener al equipo de trabajo en permanente actividad y evitar caídas de trabajo o picos de producción.

- Ayudar a interiorizar el proyecto y hacerlo suyo.

- Entrenar al equipo en labores de exposición y defensa pública del trabajo propio.

- Fomentar la competición sana entre los diferentes grupos. 
Tras la defensa preliminar del primer nivel, las encuestas de seguimiento revelaron que había un grupo que no estaba alcanzando los resultados previstos. Se reunió al grupo y se le reorientó, dándole un nuevo plazo para que pudiera corregir los resultados. Finalmente, el equipo reaccionó alcanzando una nota de 9 sobre 10 en la entrega final del trabajo.

La coevaluación también sirvió para detectar miembros del grupo cuyo nivel de implicación no estaba a la altura del resto del equipo. Tras un par de advertencias, tres alumnos fueron separados de sus equipos e invitados a continuar con el proyecto por su cuenta.

De estas personas, sólo una entregó su trabajo en solitario, no presentándose el resto. El resto de equipos finalizaron su trabajo sin incidencias.

\subsection{Encuestas}

En este apartado se presentan los resultados principales obtenidos en las encuestas finales comentadas en el punto 5.1 .

Si bien sólo el $40 \%$ de los alumnos de BB.AA. habían recibido docencia en videojuegos previamente (asignatura DiV), casi el 20\% había tenido alguna experiencia previa con Unity, y el resto no tenían ninguna experiencia. De Informática, tan sólo dos personas habían recibido docencia en videojuegos, si bien la mitad había usado previamente la herramienta a un nivel muy básico.

El hecho de disponer de más tiempo para la asignatura en BB.AA. y que la asignatura se centrara sobre todo en la integración de contenidos más que en la programación, hizo que casi el $60 \%$ de los alumnos respondieran que la frecuencia de los actos de seguimiento de la asignatura era la adecuada y el resto que era relajada.

En Informática, el 60\% también coincidió en que el ritmo de las clases era adecuado, pero a la cuarta parte le resultó demasiado intenso.

La participación en la Feria de proyectos de la ETSINF fue satisfactoria o muy satisfactoria para prácticamente todos los alumnos encuestados, siendo la respuesta de Informática casi unánimemente muy satisfactoria mientras que en BB.AA., cuyos alumnos no estaban acostumbrados a este tipo de dinámicas, se percibió más bien satisfactoria.

Por otra parte, los alumnos de Informática valoraron mejor que los de BB.AA. la utilidad de los contenidos de la asignatura de cara a dedicarse profesionalmente a la creación de videojuegos.

Los alumnos de BB.AA. valoraron positivamente la coordinación de temario entre asignaturas.

Tabla 4. Distribución de las calificaciones finales obtenidas tanto por los alumnos de BB.AA. como por los de Informática.

\begin{tabular}{ccc}
\hline Asignatura & DEV & IPV \\
\hline Matric. Honor & $1(7,14 \%)$ & $1(3,23 \%)$ \\
Sobresaliente & $3(21,43 \%)$ & $19(29,03 \%)$ \\
Notable & $6(42,86 \%)$ & $16(51,61 \%)$ \\
Aprobado & $4(28,57 \%)$ & $4(12,90 \%)$ \\
Suspenso & 0 & $1(3,23 \%)$ \\
Presentados & $14(100 \%)$ & $31(94 \%)$ \\
No presentados & 0 & $2(6 \%)$ \\
Nota media & 7,86 & 7,97 \\
Des. Estándar & 1,34 & 1,25 \\
Nota máxima & 9,5 & 9,5 \\
Nota mínima & 5,4 & 4,6
\end{tabular}

Comparando esta asignatura con el resto de asignaturas cursadas este año, el nivel de trabajo se ha percibido entre normal y alto para los alumnos de BB.AA., mientras que, para los informáticos, ha sido entre alto y muy alto, lo que está relacionado con la frecuencia de los actos de seguimiento. 
El hecho de poder evaluar a los compañeros de grupo tras cada entrega ha sido percibido por los alumnos de manera positiva o incluso muy positiva, mientras que la realimentación de los profesores en estos actos se percibió como normal.

En laTabla 4 se muestra un resumen de las calificaciones finales obtenidas por los alumnos. Como también muestra la Fig. 8 , las notas de los alumnos de DeV han sido ligeramente superiores a la de sus compañeros de IPV. Mientras que en la primera se han presentado y han aprobado todos, en la segunda ha habido un alumno suspendido y dos no presentados. La mitad de los alumnos que contestó la encuesta final obtuvo una nota mejor de lo esperado. Un $44.4 \%$ obtuvo la nota esperada, y sólo un $5.6 \%$ esperaba mejor nota.

\section{Distribución de calificaciones finales}

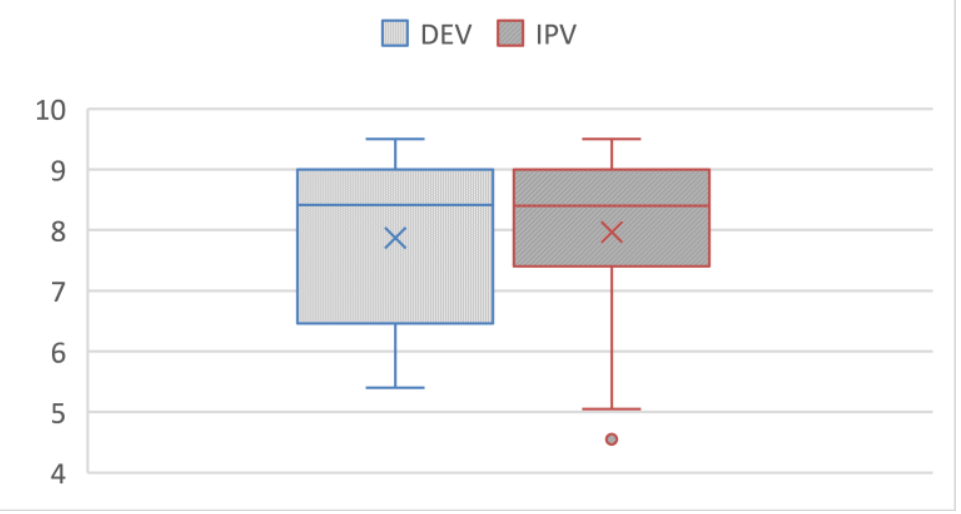

Fig. 8 Distribución de las calificaciones finales por asignatura

\section{Conclusiones}

Se ha presentado una experiencia de desarrollo multidisciplinar de videojuegos, realizada a través de la integración de dos asignaturas de dos titulaciones alejadas en sus objetivos, pero complementarias para el proyecto.

En general, los alumnos han acabado satisfechos con la asignatura. Han valorado principalmente la posibilidad que les ha brindado la experiencia para trabajar con personas de otro campo y poder realizar un videojuego completo. También han valorado muy positivamente la participación en la Feria de proyectos, para poder dar visibilidad a su trabajo, y para poder experimentar de primera mano los sentimientos que despiertan su juego en terceras personas.

A pesar de que la cantidad de actos de control y defensas realizadas han supuesto una sobrecarga de gestión para los profesores en aspectos como la votación de los mejores trabajos, la asignación de grupos, las valoraciones internas de cada grupo, organización de las defensas, etc., esto ha permitido que los proyectos alcanzaran el objetivo previsto.

Las principales quejas recibidas por parte de los alumnos se han debido a problemas de coordinación y a la diferente implicación de los miembros del grupo. En asignaturas con grupos de trabajo grandes, puede ocurrir que haya alumnos que se dejen llevar por el resto. Este problema se ha agudizado por la falta de coordinación de los horarios. El horario de las asignaturas no coincide en ningún momento, y los alumnos de cuarto tienen horarios muy distintos debido a su elección de asignaturas optativas, realización de prácticas en empresa o estar ya activos laboralmente. Para este problema, se ha buscado espacios y momentos comunes de trabajo y actos de seguimiento.

Otra queja de los alumnos, especialmente de los de BB.AA. es la falta de adaptación de los contenidos de la asignatura a la titulación. Algunos alumnos proponen menos diseño en Informática, y más desarrollo de contenidos e integración en BB.AA. (a costa de la programación). Sin embargo, los profesores pensamos que, aunque a la hora de realizar el proyecto haya una especialización natural de cada miembro del equipo, el objetivo último es que todos sean capaces de completar un videojuego sencillo por sí mismos.

En general, los profesores y la mayoría de los alumnos hemos acabado muy satisfechos con el resultado final. Todos los grupos han logrado el objetivo de obtener un videojuego funcional, que en varios casos va a evolucionar a uno o varios TFG. Es indiscutible que la calidad de los resultados obtenidos es muy 
superior a los resultados de asignaturas de otros años, en los que han participado únicamente alumnos de una misma titulación.

\section{Agradecimientos}

Este trabajo ha sido financiado por el Vicerrectorado de Estudios, Calidad y Acreditación de la Universitat Politècnica de València, a través de la Convocatoria A+D. Proyectos de Innovación y Mejora Educativa (PIME 19-20/167).

\section{Referencias}

Aalto. $<$ https://www.aalto.fi/en/aalto-media-lab/studies $>$ [Consulta: 21 de febrero de 2020]

AIE. $<$ http://www.aie.edu.au/> [Consulta: 21 de febrero de 2020]

ALARCÓN GARCÍA, G. y GUIRAO MIRÓN, C. (2014). "El enfoque de las capacidades y las competencias transversales en el EEES" en Historia y Comunicación Social, vol. 18, p. 145-157, ISSN: 1137-0734, http://dx.doi.org/10.5209/rev_HICS.2013.v18.44318

ALVAREZ BLANCO, S. et al. (2016). "Estudio de la Implantación de diversas Competencias Transversales en Asignaturas de diferentes Titulaciones de Ingeniería de la UPV" en II Congreso nacional de innovación educativa y docencia en red, IN-RED, ISBN 978-84-9048-541-5, http://hdl.handle.net/10251/105295

AS. <http://www.autograf-design.com/en/training-and-qualifications/video-game-art-3d/graphic-designart-director-video-game-creation-option/programme.html> [Consulta: 21 de febrero de 2020]

BLANCO, J.M. et al. (2014). "Diseño de una propuesta de proyecto transversal para la especialidad de Ingeniería del Software del Grado en Ingeniería Informática" en Actas de las XXI Jornadas de Enseñanza Universitaria de Informática. Oviedo: Jenui. 10-17. ISBN: 978-99920-70-09-3, http://hdl.handle.net/2117/77482

CASTRO, S.J. et al. (2018). "An Undergraduate Project combining Computer Science and the Arts: An Experience Report of a Multidisciplinary Capstone Design" en Proceedings of the 7th Computer Science Education Research Conference, CSERC '18, pp. 1-8. San Petersburgo, Federación Rusa. ACM. DOI: https://doi.org/10.1145/3289406.3289407

CAZORLA, D. et al. (2010). "Plan de coordinación docente en el Grado de Ingeniería Informática" en Actas de las XVI Jornadas de Enseñanza Universitaria de Informática. Santiago de Compostela: Jenui. 138-144. http://hdl.handle.net/2099/11770

Champlain.

$<$ https://www.champlain.edu/academics/undergraduate-academics/majors-andspecializations/game-design> [Consulta: 21 de febrero de 2020]

CHRISTOPOULOU, E. y XINOGALOS, S. (2017). "Overview and Comparative Analysis of Game Engines for Desktop and Mobile Devices" en International Journal of Serious Games, vol. 4, Issue 4, p. 21. ISSN: 2384-8766, http://dx.doi.org/10.17083/ijsg.v4i4.19

CMU. <https://ideate.cmu.edu/undergraduate-programs/game-design/> [Consulta: 21 de febrero de 2020]

CUADRADO, M., RUIZ MOLINA, M.E. y COCA, M. (2009). "Participación y rendimiento del estudiante universitario en un proyecto docente interdisciplinar, bilingüe y virtual" en Revista de Educación, vol. 348, p. 505-518. ISSN: 0034-592X

DigiPen. $\quad<$ https://www.digipen.edu/academics/computer-science-degrees/bs-in-computer-science-andgame-design $>$ [Consulta: 21 de febrero de 2020]

DU. <https://drexel.edu/westphal/academics/undergraduate/GDAP/> [Consulta: 21 de febrero de 2020]

FRODEMAN, R., THOMPSON KLEIN, J., y DOS SANTOS PACHECO, R.C. (2017). The Oxford Handbook of Interdisciplinarity, Ed. 2, Oxford: Oxford University Press. ISBN: 0191053260, 9780191053269

Gamasutra.

$<$ https://www.gamasutra.com/blogs/MarcusToftedahl/20190930/350830/Which_are_the_most_commonly _used_Game_Engines.php o bien en https://itch.io/game-development/engines/most-projects verificados el 08/02/2020> [Consulta: 21 de febrero de 2020] 
KESSLER, R., VAN LANGEVELD, M. y ALTIZER, R. (2009). "Entertainment arts and engineering”en ACM Technical Symposium on Computing Science Education SIGCSE, vol. 41, issue 1, p. 539-543. DOI: https://doi.org/10.1145/1539024.1509049

MIT. <http://gamelab.mit.edu/study/> [Consulta: 21 de febrero de 2020]

NS. $<$ https://www.noroff.no/en/studies/vocational-school/3d-game-design $>$ [Consulta: 21 de febrero de 2020]

Oulu. $<$ http://www.oulugamelab.net/overview $>$ [Consulta: 21 de febrero de 2020]

PARKER, J.R. (2004). "Games and animation: collaborations between the arts and computer science" en International Conference on Information Technology: Coding and Computing, ITCC. Las Vegas. IEEE, DOI: 10.1109/ITCC.2004.1286444, ISBN: 0-7695-2108-8

PEÑA PAZ, L. et al. (2011). "Proyecto transversal del ciclo básico de ingeniería: una experiencia de articulación curricular desde las competencias" en Ninth LACCEI Latin American and Caribbean Conference (LACCEI'2011). Medellín. ISBN 978-0-9822896-4-8

RIES, E. (2011). The Lean Startup. New York: Crown Publishing Group, ISBN-10: 9780307887894

RIT. <https://www.rit.edu/study/game-design-and-development-bs $>$ [Consulta: 21 de febrero de 2020]

SÁNCHEZ-ELVIRA PANIGUA, Á., LÓPEZ-GONZÁLEZ, M. Á. y FERNÁNDEZ-SÁNCHEZ, M. V. (2010). "Análisis de las competencias genéricas en los nuevos títulos de grado del eees en las universidades españolas" en REDU. Revista de Docencia Universitaria, vol. 8, issue 1, p. 35-73. https://doi.org/10.4995/redu.2010.6217

SCAD. $\quad<$ https://www.scad.edu/academics/programs/interactive-design-and-game-development $>$ [Consulta: 21 de febrero de 2020]

SERRANO TIERZ, A. el al. (2013). "Trabajo por módulos: un modelo de aprendizaje interdisciplinar y colaborativo en el Grado en Ingeniería en Diseño Industrial y Desarrollo de Producto" en REDU Revista de Docencia Universitaria, vol. 11, p. 97-220, ISSN:1887-4592, https://doi.org/10.4995/redu.2013.5553

Sheridan. $<$ https://academics.sheridancollege.ca/programs/bachelor-of-game-design $>$ [Consulta: 21 de febrero de 2020]

TFZ. <http://www.torontofilmschool.ca/programs/video-game-design-development/> [Consulta: 21 de febrero de 2020]

Unity. $<$ https://unity.com/es $>$ [Consulta: 21 de febrero de 2020]

USC. $<$ https://games.usc.edu/> [Consulta: 21 de febrero de 2020]

VCAD. <http://www.vcad.ca/programs/game-development-and-design/> [Consulta: 21 de febrero de 2020]

VERNET, D., CANALETA, X. y PLANAS J. (2011). "LSMaker: un proyecto interdisciplinar" en Actas de las XVII Jornadas de Enseñanza Universitaria de Informática, Jenui 2011. Sevilla: Jenui. 13-20. ISBN: 978-84-694-5440-4

VFS. $<$ https://vfs.edu/programs/game-design> [Consulta: 21 de febrero de 2020]

WOOD, Z.J., MUHL, P, y HICKS, K. (2016). "Computational Art: Introducing High School Students to Computing via Art" en Proceedings of the 47th ACM Technical Symposium on Computing Science Education, SIGCSE '16. Tennessee, USA. p. 261-266. ISBN: 978-1-4503-3685-7, DOI: https://doi.org/10.1145/2839509.2844614 